\title{
Results Of Localized Alfvén Wave Heating In TCABR
}

\author{
A. G. Elfimov, E. A. Lerche, R. M. O. Galvão, L. F. Ruchko, \\ A. M. M. Fonseca, R. P. da Silva, and V. Bellintani \\ Departamento de Física Aplicada, Instituto de Física, \\ Universidade de São Paulo, 05315-970, São Paulo, SP, Brazil
}

Received on 2 March, 2004; revised version received on 24 April, 2004

\begin{abstract}
Using two dimensional numerical codes, we show that the direct electron heating found with electron cyclotron emission (ECE) radiometry is related to local Alfvén wave resonances in Tokamak Chauffage Alfvén Brésilean (TCABR) plasmas. The $m / N=1 / 2,0 / 2$ and $0 / 3$ modes are dominant in the observed wave heating, due to Alfvén wave continuum absorption. In particular, the resonant absorption of the $m=0$ sideband harmonic plays an important role in the central core and peripheral heating. The increase of the electron temperature during the radio frequency pulse was accompanied by a local density rise that produced a cut-off of the ECE emission. The observed heating profile is also consistent with the reflectometer measurements of the density fluctuations induced by radio frequency fields in the local Alfvén wave resonance of the $m / N=-1 /-2,1 /-3$ modes in TCABR.
\end{abstract}

\section{Introduction}

In early studies, Alfvén waves (AW) were proposed (see, for example, [1]) as an alternative to cyclotron heating in the ion cyclotron range of frequencies (ICRF) and current drive by fast waves in large tokamaks because of the availability of high power radio-frequency (RF) generators, the simplicity of wave launching structures and the lack of the plasma density limit for current drive (in comparison with lower-hybrid waves). Recently, improvement of the energy confinement was achieved with internal transport barriers (ITB) created by neutral beam injection (NBI) and by wave heating in the ion cyclotron range of frequencies (ICRF) in all large tokamaks like Doublet-III-D, Joint European Torus (JET) and others (see, for example, review [2]). According to the theory, ITB appear in tokamaks because of the current profile modification (negative shear) or strongly sheared poloidal rotation [3]. In this line of study, AW are currently being considered as a tool to control current profile and to drive the locally sheared plasma flow in tokamaks [4] because they can deposit energy mainly at the local Alfvén resonance. The idea of heating magnetically confined plasmas by the resonant absorption of RF fields using the local AW resonance began with the theoretical work carried out by Dolgopolov and Stepanov [5], Grossmann and Tataronis [6], and Hasegawa and Chen [7]. AW heating is based upon mode conversion of an externally driven RF field below the ion-cyclotron frequency, $\omega_{c i}$, into the kinetic or electrostatic Alfvén wave (shear AW) at the Alfvén resonance layer. In the standard cylindrical model for wave excitation in tokamak plasmas, the oscillating RF field is represented as a sum of helical harmonics $\exp \left[\mathrm{i}\left(m \theta+N z / R_{0}-\omega t\right)\right]$, where $m$ is the poloidal and $N$ is the toroidal (axial) wave numbers that are excited in the plasma by an antenna current sheet,

$j_{\theta, \phi}=\sum_{M, N} J_{\theta, \phi}(M, N) \delta(r-b) \exp \left[\mathrm{i}\left(M \theta+N z / R_{0}-\omega t\right)\right]$

Here, we note that, due to toroidal effects, the poloidal wave numbers $m$ in the plasma may be different from those excited by the antenna. The local AW resonance appears at the radius $r$ where the frequency of the RF field driven by an external antenna with axial $N$ and poloidal $m$ wavenumbers matches with AW frequency corresponding to the equation,

$$
k_{\|}^{2}=\frac{\omega^{2}}{c^{2}} \epsilon_{\perp} \Rightarrow k_{\|}^{2}=\frac{\omega^{2}}{c_{A H}^{2}}\left[\frac{n_{i} / n_{e}}{1-\omega^{2} / \omega_{i}^{2}}+\frac{A_{z} n_{z} / n_{e}}{1-\omega^{2} / \omega_{z}^{2}}\right]
$$

where $k_{\|}=\left(N / R_{0}\right)(1+m / N q) B_{\zeta} / B$ is the parallel component of the wavevector, $q=\left(r / R_{0}\right) B_{\zeta} / B_{\theta}$ is tokamak safety factor, $c_{A H}(r)=B / \sqrt{4 \pi m_{H} n_{e}(r)}$ is the Alfvén velocity in the hydrogen plasma, $B_{\theta, \zeta}$ are the poloidal and toroidal components of the magnetic field $B$, and $R_{0}$ is the major radius of the toroidal plasma column of minor radius $a$. We note that the impurities with the same mass/charge ratio and the mass number $A_{z}$ and density $n_{z}$ are included into AW continuum dispersion in eq.(1). This dispersion relation gives the local $\mathrm{AW}$ velocity $c_{A}=\sqrt{A_{H} / A_{\mathrm{ef}}} c_{A H}$ in low frequency limit where $A_{\text {ef }}=\sum_{i} A_{i} n_{i} / n_{e}$. The continuous spectrum of these local resonance frequencies (1) is well known as the Alfvén wave continuum [6].

In the AW continuum, when the electron thermal velocity is larger then the local AW velocity $\left(v_{T e}>c_{A}\right)$, the kinetic Alfvén wave (KAW) is excited at the mode conversion surface, propagating towards the plasma center [7], and its Landau and/or collisional damping leads to electron heating. When $v_{T e}<c_{A}$, a slow quasi-electrostatic Alfvén wave (SQEAW) is excited with mainly collision damping 
and propagates towards the plasma boundary. The detailed picture of resonant absorption was established by subsequent theoretical work carried out by different investigators (see, for example, [8]-[13]) and experimentally confirmed in tokamaks [14]-[17] and stellarator [1, 18].

Furthermore, it was shown by Ross et al [9] and Appert et al [10] that AW can also be excited in a plasma column as global modes, i.e., their wave fields have a global structure corresponding to the discrete eigenfunctions of the proper boundary value problem. The eigenfrequencies of these global Alfvén waves (GAW) lie just below the corresponding Alfvén continua. Their existence was experimentally confirmed in the Tokamak Chauffage Alfvén (TCA), PRETEXT and Phaedrus-T tokamaks $[15,16,17]$. Easy identification of GAW resonance with magnetic probes and its close position to the AW threshold minimum $\left(\Delta \omega_{G W} \ll \omega_{A, \min }\right)$ calculated from eq.(1) was explored, as diagnostic tools (named Magneto-Hydro-Dynamic diagnostics) for definition of the effective ion mass number $A_{\mathrm{ef}}=\sum_{i} A_{i} n_{i} / n_{e}$ and q-profiles in TCA, TEXTOR, JET tokamak experiments $[22,23,24]$. However, the strong dependance of $\Delta \omega_{G W}$ on toroidal wave number and current and density profile produced substantial difficulties in the interpretation of the experimental data.

The other difficulty of AW theory is toroidal effects, which can substantially modify the picture of Alfvén wave absorption in tokamaks. In toroidal geometry, the theory predicts $[10,13,20]$ that a set of different poloidal modes $m=M \pm 1, \pm 2 \ldots$ (different from antenna modes $M$ ) can be exited because of the poloidal inhomogeneity of the toroidal magnetic field $B$ in the tokamak plasmas. Although the absorption peaks of GAW are well predicted by the cylindrical model, each global mode can now appear inside the continua of other toroidally coupled modes, bringing in toroidicity induced continuum damping, with damping rate much higher than predicted by kinetic cylindrical codes, and the power deposition profiles calculated with the kinetic toroidal model [20] becomes much broader than in the cylindrical case. To calculate the position of local AW resonances and the absorbtion profile in tokamaks, a multi (electron and ion) fluid toroidal code (ALTOK) was developed [21], where collisional dissipation is taken into account, which can also be useful in the conditions of cold plasmas $\left(v_{T e}<c_{A}\right)$. The toroidal effects were already verified in previous TCA experiments in Lausanne, where density fluctuations in the local AW resonance of the sideband harmonics exited by the GAW were detected by laser interferometry [19].

The TCA tokamak was transferred to the Plasma Laboratory of the University of São Paulo and renamed Tokamak Chauffage Alfvén Brésilien (TCABR). A complete new antenna was designed and installed in the device [25], and some results (with and without Faraday screen) of AW heating and current drive (without any profile measurements) were reported [26, 27, 28]. Therefore, it is mandatory to investigate theoretically and experimentally the AW power deposition and RF field profiles for the plasma parameters of TCABR with the new antenna. Recently, using electron cyclotron emission (ECE) radiometry, a direct demonstration of local AW electron heating with the modernized antenna was achieved in TCABR tokamak plasmas [29]. In appro- ximately the same conditions, direct density fluctuations at the local AW resonance were detected using a simple reflectometer [30].

In this paper, we present results of the calculations with two dimensional codes $[20,21]$ related to the last results of localized AW heating $[29,30]$. We show that combination of small AW power deposition in local AW resonances swaped by density variation (or scanned with AW frequency), in combination with detection of density fluctuations by reflectometer in the local AW resonance, can serve as diagnostic tools for identification of the effective ion mass number $A_{\mathrm{ef}}$ and q-profile in tokamaks. The idea is based upon the simultaneous detection of the position of $m=0$ and $m= \pm 1$ local AW resonances that are excited by $M / N=1 / 2,1 / 3$ antenna modes. According to eq. (1), the $m=0$ resonance depends only on the effective ion mass number and does not depend on q-profile. Then, using this data we can define qvalue at the position of $m= \pm 1$ local AW resonances. The proposed method is most efficient in the low frequency band $\omega \ll \omega_{c i}$.

\section{Parameters of TCABR and Plasma Model}

The calculations with codes [20, 21] have been carried out assuming circular cross-section tokamak geometry. To obtain good accuracy, five poloidal harmonics ( $m=M \pm$ $1, \pm 2$ ) are used in the kinetic code and $263 \times 59$ mesh points are used in ALTOK code calculations. Here, we analyze the AW heating experiments $[29,30]$ in TCABR tokamak (minor radius a $=0.18 \mathrm{~m}$, major radius $R_{0}=0.615 \mathrm{~m}$, toroidal magnetic field $\mathrm{B}=1.15 \mathrm{~T}$ ) using the following basic plasma parameters: current $I_{P}=72-92 \mathrm{kA}$, with respective edge safety factor $q(a) \simeq 3.2-3.8$; line averaged plasma density $\bar{n}=(1-2) \times 10^{19} \mathrm{~m}^{-3}$; central electron and ion temperatures $500 \mathrm{eV}$ and $150 \mathrm{eV}$, respectively; the working gas was hydrogen. The RF power delivered by the antenna was limited to $P_{R F} \leq 50 \mathrm{~kW}$ and the generator frequency was fixed at $f=4.0 \mathrm{MHz}$. The antenna module has two groups of RF current carrying straps. These groups are positioned in two toroidal cross-sections separated toroidally by an angle about $22^{0}$ creating mainly the spectrum of the poloidal $M=$ \pm 1 and toroidal $N= \pm 1, \pm 2, \pm 3$ modes.

To model the plasma conditions of TCABR in the calculations of the RF fields with the kinetic code [20], we assume simple cylindrical fitting of plasma parameter profiles; parabolic temperature profile $T_{e, i}=T_{e, i_{0}}\left[1-(r / a)^{2}\right]^{2}+T_{e, i_{a}}$ with the pedestal electron and ion temperatures $T_{e_{a}}=20 \mathrm{eV}$ and $T_{i_{a}}=15 \mathrm{eV}$, respectively. The electron density profile is given by $n_{e}=n_{0}\left[\left(1-\left(1-n_{a} / n_{0}\right)(r / a)^{2}\right)\right]$ with $n_{0}=1.7-2.5 \times 10^{19} \mathrm{~m}^{-3}$ and $n_{a}=2 \times 10^{18} \mathrm{~m}^{-3}$. The ion density $n_{i}$ is taken to satisfy the requirement of charge neutrality, $\sum_{i} Z_{i} n_{i}=n_{e}$. Using experimental data that will be discussed in the sequel for $I_{P}=89 \mathrm{kA}$ $\left(q_{a}=3.4\right)$, with safety factor $q_{0}=0.97$ as indicated by saw teeth oscillations, we chose the respective current profile $j=j_{0}\left[1-(r / a)^{2}\right]^{2.4}$. The plasma profiles used in ALTOK code are $n=n_{0}\left(1-\Psi^{0.7}\right)$ and $j=j_{0}\left(1-\Psi^{0.85}\right)^{1.4}$, where $\Psi$ is the poloidal magnetic flux), which are slightly 
different from those of the cylindrical plasma model. This difference appears because of the Shafranov shift and toroidicity effects. To explain better the experimental data [29], we assume that average effective mass number $A_{\text {ef }}$ is 1.2 in accordance with $Z_{\text {ef }} \approx 1.6$ found from ASTRA transport code calculations. It means that the AW frequencies should be diminished by $10 \%$ in comparison with the ones obtained for pure hydrogen plasmas. In Fig. 1a,b,c, we show the distribution of the q-parameter and equilibrium density, the $m=0,1, N=2$ AW continuum frequency, and the respective distribution of absorbed power obtained with the kinetic and fluid codes. The RF fields are excited by the $M / N=-1 /-2$ antenna with $4 \mathrm{MHz}$ frequency in AW continuum $\left(q_{0}=0.97, n_{0}=2.1 \times 10^{19} \mathrm{~m}^{-3}\right)$. Low electron temperature $\left(T_{e}=400 \mathrm{eV}\right)$ is used to show QESAW excitation at the plasma boundary. In Fig. 1c, we can observe very good similarity in the distribution of absorbed power for both code calculations.

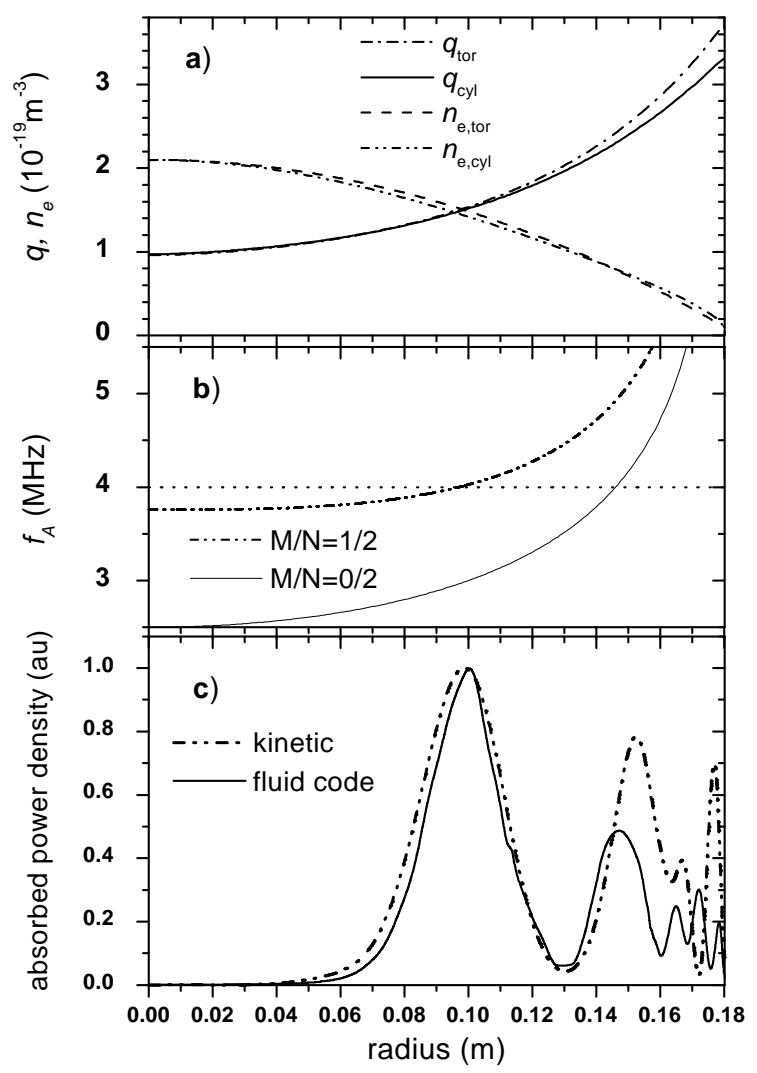

Figure 1. Plot of the density and q-profiles (a), Alfvén wave continuum frequency for $m / N=-1 /-2$ and $0 /-3$ modes (b), and respective distribution of absorbed power calculated with kinetic and fluid codes (c).

\section{Alfvén wave Absorbtion and Fields in TCABR}

To begin the discussion of AW absorbtion in TCABR, we calculate the antenna impedance using the kinetic code for poloidal $M=-1$ and toroidal $N=-2,-3$ modes for the central density variation in the interval $(1.75-2.1) \times 10^{19}$ $\mathrm{m}^{-3}$, giving line averaged densities $(1.2-1.4) \times 10^{19} \mathrm{~m}^{-3}$ in the TCABR experiments [29]. We calculate the antenna impedance [9] $Z_{a}=W / I_{\mathrm{an}}^{2}$, where $W$ is total value of the AW absorbed power and $I_{\mathrm{an}}$ is antenna current. In Fig. 2, we show the antenna impedance for $m / N=-1 /-2,0 /-3$ plasma modes excited with $4 \mathrm{MHz}$ frequency. We can observe that AW continuum absorbtion begins from $n_{0} \approx 1.95 \times 10^{19}$ $\mathrm{m}^{-3}$ when the density grows. We stress our attention on this point because a density rise usually happens during AW heating [15, 29].

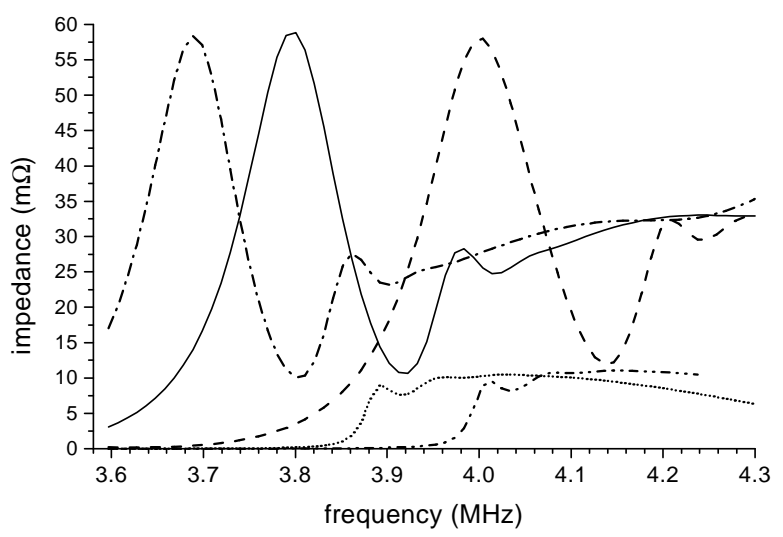

Figure 2. The impedance for the $M / N=-1 /-2$ antenna (dashed line $n_{0}=1.75 \times 10^{19} \mathrm{~m}^{-3}$, solid line $n_{0}=1.95 \times 10^{19} \mathrm{~m}^{-3}$, dashdot line $n_{0}=2.1 \times 10^{19} \mathrm{~m}^{-3}$ ), and for $-1 /-3$ antenna (dash-dot-dot line $n_{0}=1.95 \times 10^{19} \mathrm{~m}^{-3}$, doted line $n_{0}=2.1 \times 10^{19} \mathrm{~m}^{-3}$ ) calculated with kinetic code $\left(A_{\mathrm{ef}}=1.2\right)$.

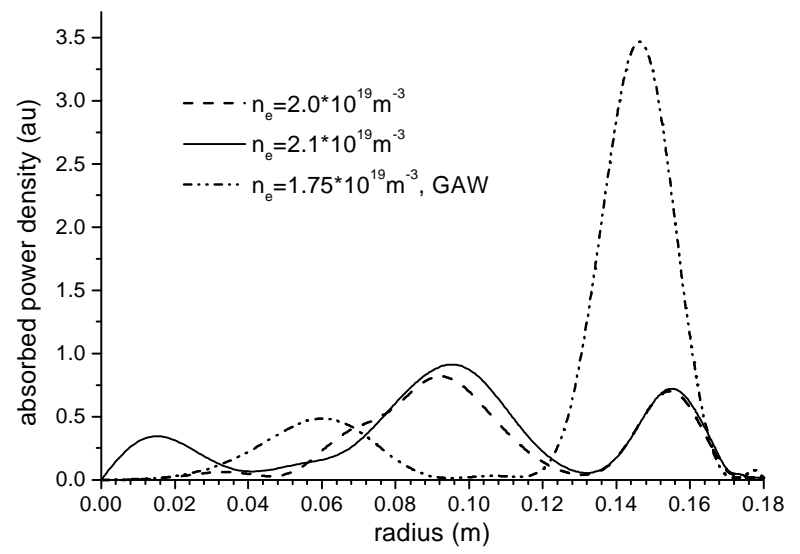

Figure 3. Plot of the power deposition profile for $4 \mathrm{MHz}$ frequency and $M / N=-1 /-2$ antenna for $n_{0}=1.75 \times 10^{19} \mathrm{~m}^{-3}$, $n_{0}=2 \times 10^{19} \mathrm{~m}^{-3}, n_{0}=2.15 \times 10^{19} \mathrm{~m}^{-3}$ (the other plasma parameters are the same as in Fig. 2).

In Fig. 3, we present the power deposition profiles for three central densities $\left(n_{0}=1.75 \times 10^{19} \mathrm{~m}^{-3}, n_{0}=2 \times 10^{19}\right.$ $\left.\mathrm{m}^{-3}, n_{0}=2.15 \times 10^{19} \mathrm{~m}^{-3}\right)$, respectively, in GAW resonance, at the edge and in the depth of the AW continuum. The respective resonance conditions for $\mathrm{f}=4 \mathrm{MHz}$ are shown in Fig. 2. The combinations of $m / N=-1 /-2,-1 /-3$ antenna modes with $4 \mathrm{MHz}$ frequency is assumed in these calculations. We can observe that the main power deposition in the GAW resonance is moved from the plasma core, predicted by the cylindrical model, to the sideband $(m / N=0 /-2)$ 
local AW resonance region. Analyzing the power distribution in Fig. 3, we find that the best results for the plasma core heating is expected for the conditions of deep AW continua when, together with $m / N=1 / 2$ continuum, it appears the $m / N=0 /-3$ continuum absorbtion, as it shown in Fig. 2 . In this case, the $m / N=0 /-3$ local AW resonance is excited by $M / N=1 /-3$ antenna in the plasma core.

\section{Discussion of Experimental Results}

In this section, we discuss the experimental measurements of radiative electron temperature [29] in TCABR with Electron Cyclotron Emission radiometry (ELVA-1) (see reviews about ECE diagnostics in Ref.[31, 32]). Since the basic description of the radiometry used in the experiments was absent in the initial reference [29], we briefly describe ECE radiometry in Appendix.

\subsection{Results of Alfvén wave heating experi- ments}

The AW heating experiments were carried out in TCABR keeping the value of the plasma density rather low but above runaway density limit $n_{e 0}>1.5 \times 10^{19} \mathrm{~m}^{-3}$, to allow reasonable accessibility of the second-harmonic electron cyclotron emission $n_{e 0}<2.3 \times 10^{19} \mathrm{~m}^{-3}$, and to operate in a window of the AW continuum for which the resonant absorption is not spoiled by cyclotron absorption of impurity ions, such as $\mathrm{O}^{+3}$ and $\mathrm{O}^{+4}\left(\right.$ or $\mathrm{C}^{+3}$ ) at the plasma edge. Here, we use the data from a typical time evolution plot of the plasma parameters (loop voltage, current, central line averaged density, bolometry and soft X-ray signals) for TCABR shot 9796 presented in [29]. The RF pulse of $50 \mathrm{~kW}$ power delivered by antenna begins at $t=75 \mathrm{~ms}$ with the pulse duration about $10 \mathrm{~ms}$ during flat current $\left(I_{p}=88 \mathrm{kA}\right)$ stage when the monotonic density rise about $10-15 \%$ is observed.

To get good time resolution of the relative electron temperature increase during the RF pulse, the electron temperature is measured in only one radial position, displacing it on a shot-to-shot basis. Results of ECE time traces for different radial positions (with other TCABR parameters similar of those to shot 9796) are presented in Fig. 4, where the measured radiative temperature, $T_{e}$, is normalized to its value $<T_{e}>$ averaged over $5 \mathrm{~ms}$ before the application of the RF pulse. Sawtooth oscillations can be seen in some of the ECE traces. These oscillations have a period $\tau_{\text {saw }}=0.44$ $\mathrm{ms}$, and the inversion radius $(q=1)$ occurs near $R=0.66$ $\mathrm{m}$, approximately $4 \mathrm{~cm}$ from the magnetic axis. This information together with the boundary value $q_{a}=3.4$, allows a reasonable fitting of the $q(r)$ profile to use in the simulations of the AW absorption, discussed in Sect.3. During the RF pulse, the temperature increased in all radial positions shown in Fig. 4, except in the layer between $R=0.65 \mathrm{~m}$ and $R=0.68 \mathrm{~m}$, where the temperature dropped. This layer corresponds to the already referred ECE cut-off region that is just barely touched for a density $n_{e 0} \approx 2.2 \times 10^{19} \mathrm{~m}^{-3}$, and not fully achieved over the whole bandwidth of the ECE radiometer.
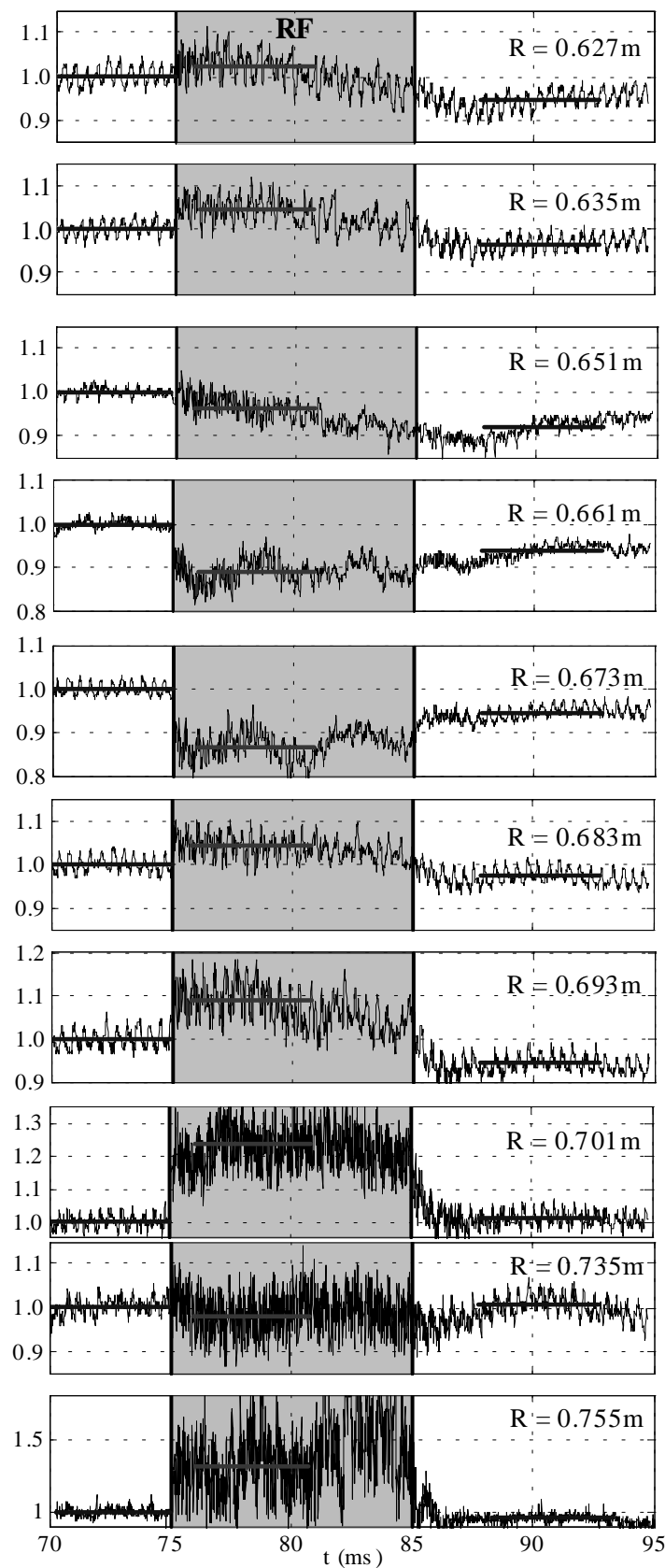

Figure 4. Time traces of the radiative electron temperature measured by the ECE radiometer over different chords, which are normalized to respective temperatures before $\mathrm{RF}$ pulse $\left(T_{e} /<T_{e}>\right)$ in TCABR.

To get a better picture of the power deposition profile, in Fig. 5a, we plot the relative temperature increase $\Delta T_{e} /<T_{e}>$, as a function of the normalized minor radius $\left(R-R_{0}\right) / R_{0}$. The agreement between the relative temperature variation and the calculated power absorption profiles shown in Fig. 5b is surprisingly good. As we expected, most of the heating occurred at the middle of the plasma minor radius, where resonant absorption takes place in the main local AW resonance $(m / N=-1 /-2)$. The temperature increase observed in the plasma center is consistent with the absorbtion of the continuum side-band harmonic $(m / N=0 /-3)$. The to- 
roidicity induced $0 /-2$ local AW resonance is responsible for most of the undesired power deposition near the plasma border. Comparing the discussed power deposition with calculated one for the GAW resonance shown in Fig. 3, we can conclude that the GAW power deposition is not consistent with the electron temperature variations except in the region near plasma border $(m / N=0 /-2$ local AW resonance).
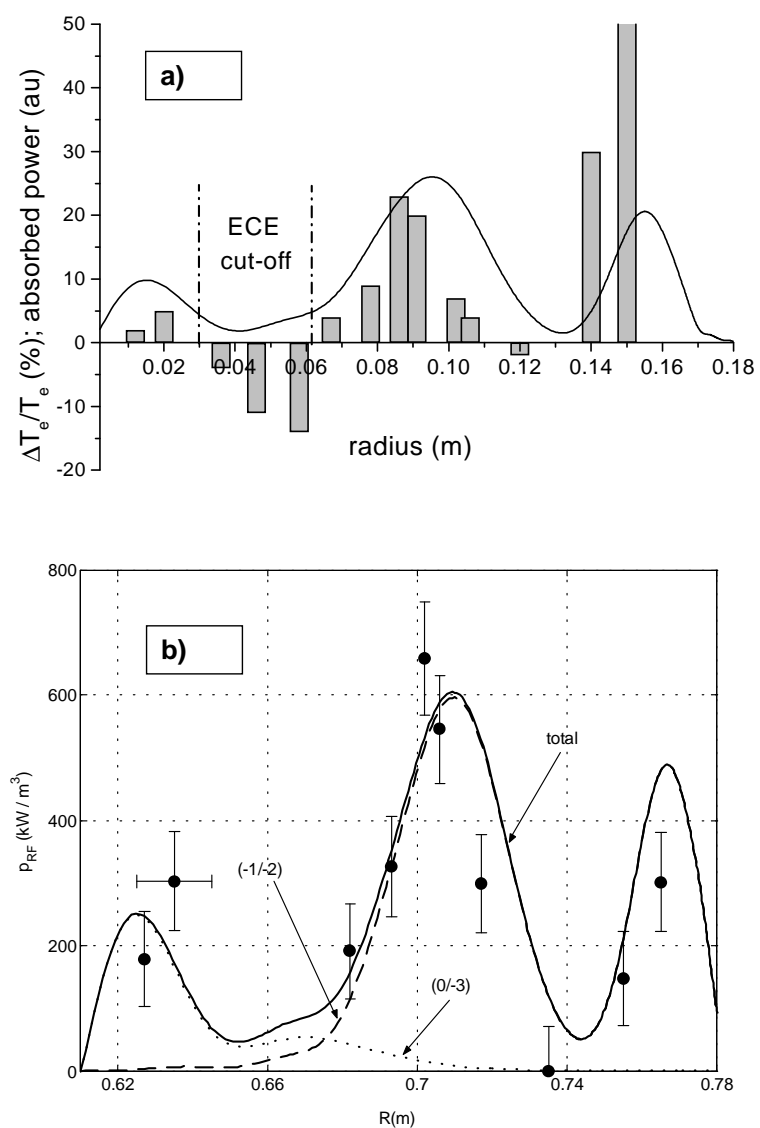

Figure 5. Plot of the distribution of the normalized radiative electron temperature taken from Fig. 4 at the beginning of the RF pulse and the respective distribution of absorbed AW power calculated with kinetic code for $M=-1$ and $N=-3,-2$ antenna (a); the absorbed AW power distribution (black circles) calculated from the experimental data in Fig. 4a in the comparison with the kinetic code calculations (b).

To check the consistency of the observed temperature variation with AW heating, we have estimated the total heating power delivered by the antenna and compared the result with the measured RF absorbed power. The average heating power was estimated using the equation $P_{R F}=<n_{e}><$ $\Delta T_{e} / \tau_{R F}>$. In this expression, $\left\langle n_{e}>\right.$ is the average plasma density in the layer of volume $\delta V$ and $\Delta T_{e}$ is the mean electron temperature variation caused by the wave absorbtion. Evaluating the average characteristic RF heating time from the initial temperature rise shown in Fig. 4, we obtained $\tau_{R F} \approx 200 \mu \mathrm{s}$. The values of the estimated heating powers shown in Fig. $5 \mathrm{~b}$ are in a good agreement with code calculated absorption in these regions $(3,19$ and 12 $\mathrm{kW}$, respectively). For the three regions shown in Fig. 5, the total calculated power is about $34 \mathrm{~kW}$, which is smaller than the total delivered power $(50 \mathrm{~kW})$. However, since the power coupled into the radial layer with ECE cut-off and that coupled parasitically to the plasma edge were not accounted for, this estimate is at least consistent with the claim of direct AW heating. The loop voltage variation was similar (or slightly higher) to ohmic discharges without RF heating, i.e., there was no loop voltage decrease corresponding to heating and current drive [17]. To make the results of transport simulations carried out with the ASTRA code consistent with the observed heating and time evolution of the loop voltage, we have to increase the value of the effective charge number $Z_{\text {ef }}=1.6$ by $10-15 \%$, produced by fully ionized carbon or oxygen impurities, what corresponds to a variation of the effective mass number $A_{\text {ef }}$ from 1.2 to 1.3. We note that the experimental error is about $2-3 \mathrm{~cm}$ in the position of the radiative temperature maxima found with ECE radiometer, which is too large to help in identification of q-values in the local AW resonances.

\subsection{Identification of Local AW Resonance with Reflectometer}

As was shown by Ruchko and co-authors [30], the density fluctuation rise in the AW power deposition regions can be detected by a reflectometer with the fixed frequency (32.4 $\mathrm{GHz}$ ). The detection principle is based on the reflection of the $O$-mode field from the cut off layer density defined by the condition $n_{\mathrm{ctf}}=\pi f_{\mathrm{ref}}^{2} m_{e} / e^{2}$. For a small density perturbation presented in the one dimensional form, $\delta n_{e} \cos \left[k_{r}\left(r-r_{c}\right)\right]$ ( $r_{c}$ is the location of the cutoff layer), a simple expression for the perturbed phase $\delta \phi$ can be used,

$$
\delta \phi \approx 2 k_{0} \sqrt{L_{n} / k_{r}} \delta n_{e} / n_{e}, \quad L_{n} k_{r}>1
$$

where $L_{n}$ is the density scale length at the cutoff layer, $k_{r}$ is the wave number of the density perturbation in the direction of wave propagation. In hot plasmas $c_{A}<v_{T e}$, using the results of Ref. [9] the amplitude of the density fluctuations in the local AW resonance is defined by the simple equations,

$$
\frac{\left|\delta n_{e}\right|}{n_{e}}=\frac{e\left|\tilde{E}_{\|}\right|}{m_{e} k_{\|} v_{T e}^{2}},\left|\tilde{E}_{\|}\right|=2 \sqrt{\frac{\sqrt{8 \pi} k_{\|}^{3} v_{T e}^{3}}{\pi \omega_{p e}^{2} \omega_{\mathrm{ref}}^{2}} \tilde{p}}
$$

where $\tilde{p}$ is the absorbed power density. In Fig. 6, we show the time traces (70-100 ms) of the loop voltage, line averaged density, toroidal current, and respective amplitude of oscillating phase measured with the reflectometer in discharge \# 10668, with AW heating [30]. The perturbed density trace during RF pulse is restored from bolometry signal $\sim Z_{\text {eff }}^{4} n_{e}^{2} / T_{e}^{3 / 2}$, assuming that there is small $Z_{\text {eff }}$ rise from 1.6 to 1.8 , as found from ASTRA calculations using the small loop voltage increase $\left(\sim Z_{\text {eff }} / T_{e}^{3 / 2}\right)$ at the beginning of the RF pulse. The central electron temperature is not increased during RF pulse, as indicated by the ECE signal. We chose this shot because their plasma parameters are very similar to AW heating discharges discussed in the previous subsection, with exception of the reduced delivered power by a factor two. In Fig. 6c, we can observe the maxima of the amplitude of oscillating microwave phase $(4 \mathrm{MHz})$ that can be interpreted as local AW resonances discussed below. 


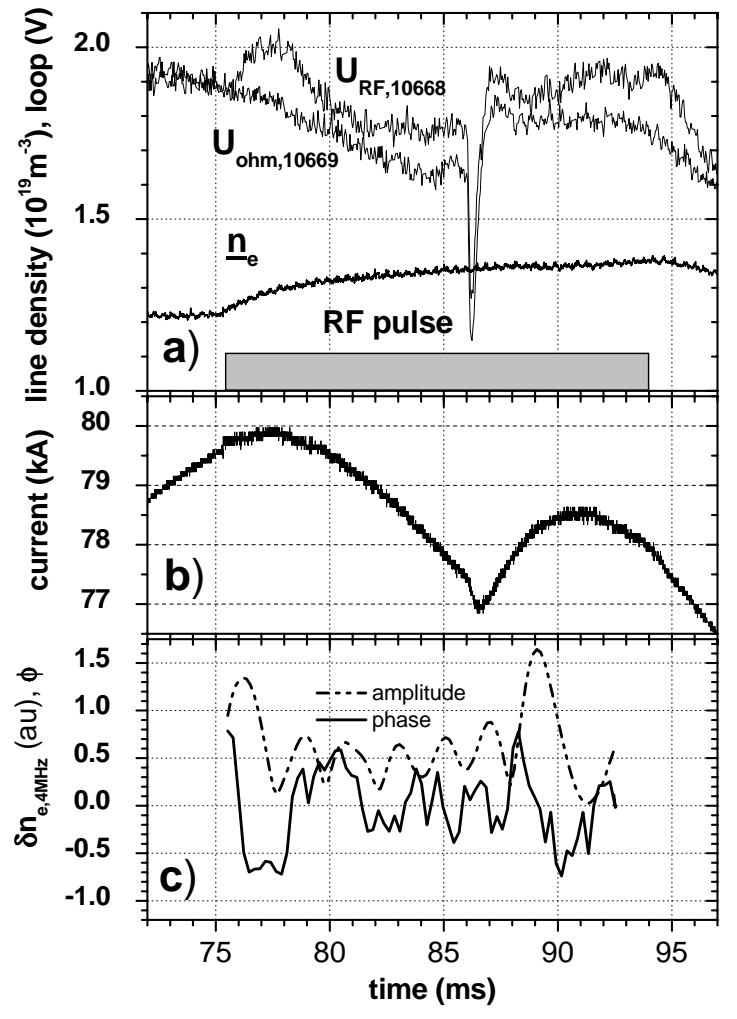

Figure 6 . The time traces (70-100 ms) of the loop voltage and line averaged density (a), toroidal current (b) and respective amplitude of oscillating phase measured with reflectometer (c) in the TCABR discharge with AW heating (\# 10668).

Generally, the RF power deposition and respective density oscillation regions are defined by the local AW resonance (1), which depends on the generator frequency $f_{R F}=$ $\omega / 2 \pi$, poloidal and toroidal wavenumbers $(m / N)$, the central plasma density, the safety parameter $q$ and effective mass number $A_{\text {ef }}$. It is convenient to rewrite eq.(1) in the form of the definition for the AW resonance density,

$$
n_{A}=n_{0} \frac{c_{A H}^{2}(0) k_{\|}^{2}(r)}{\omega^{2}}\left[\frac{n_{i} / n_{e}}{1-\omega^{2} / \omega_{i}^{2}}+\frac{A_{z} n_{z} / n_{e}}{1-\omega^{2} / \omega_{z}^{2}}\right]^{-1}
$$

The cross section of the $n_{A}$ value with the electron plasma density gives us the local AW resonance position. From eq.(3), we find that the scanning of the local AW resonance density in relation to the cut off density is possible only with growth of the impurity density $n_{z}$ or with q-parameter variations. In Fig. 7a, using the parameters of this shot we show the Alfvén $n_{A}$ (for $m / N=-1 /-2,1 /-3$ ) and $n_{e}$ electron density distributions over radius and the position the cut off density $n_{\text {ctf }}=1.29 \times 10^{19} \mathrm{~m}^{-3}$. The respective amplitudes of the density fluctuations for $4 \mathrm{MHz}$ frequency, $m / N=$ $-1 /-2,1 /-3, n_{0}=1.9 \times 10^{19} \mathrm{~m}^{-3}, n_{z} / n_{e}=1.6 \%$ and $m / N=-1 /-2, n_{0}=2.1 \times 10^{19} \mathrm{~m}^{-3}, n_{z} / n_{e}=2.4 \%$ are shown in Fig. 7b. Here, we can observe that the local AW resonance $(m / N=1 /-3)$ can be excited at the cut off density at the beginning of RF pulse and the local AW resonance $(\mathrm{m} / \mathrm{N}=-1 /-2)$ is excited at the end of RF pulse related to Fig. 6.

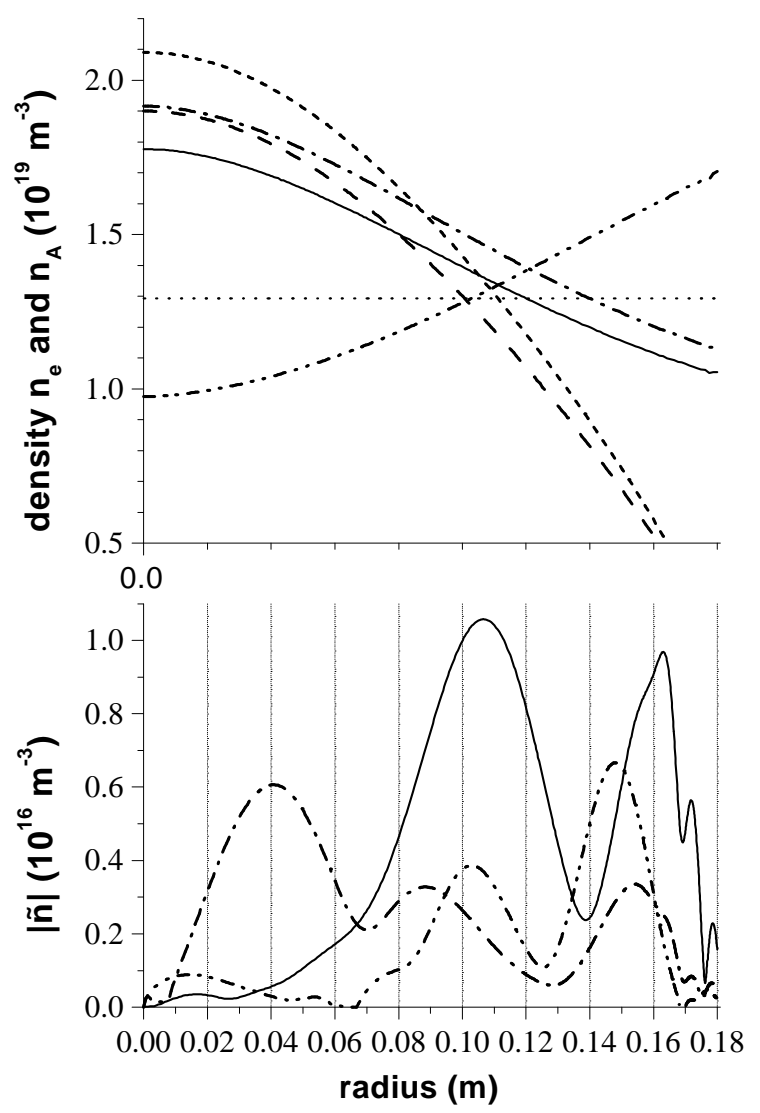

Figure 7. Plot of (a) Alfvén density distributions for $A_{\mathrm{ef}}=1.17$, $q=1.03, m / N=-1 /-2$ (dash-dot line) and $m / N=-1 / 3$ (dashdot-dot line) modes, and for $A_{\text {ef }}=1.25, q=1.07$ (solid line) over radius, the electron density distributions $\left(n_{0}=1.9 \times 10^{19} \mathrm{~m}^{-3}\right.$ dashed and $n_{0}=2.1 \times 10^{19} \mathrm{~m}^{-3}$ short dashed lines), the cut off density is marked by dotted line; (b) the amplitudes of the AW density fluctuations for $4 \mathrm{MHz}$ frequency, respective to (a), $m / N=-1 /-2$ (dash-dot line $n_{0}=1.9 \times 10^{19} \mathrm{~m}^{-3}$ and solid line $\left.n_{0}=2.1 \times 10^{19} \mathrm{~m}^{-3}\right), \mathrm{m} / \mathrm{N}=1 / \mathrm{-} 3$, (dash-dot-dot line $n_{0}=$ $1.9 \times 10^{19} \mathrm{~m}^{-3}$ ).

\section{Conclusion}

Theoretical analysis show that RF heating detected with electron cyclotron emission (ECE) radiometry is related to local AW electron power deposition in TCABR tokamak plasmas. The increase of the electron temperature during the radio frequency pulse was accompanied with the local density and $Z_{\text {eff }}$ rise that produces an cut-off of the ECE emission. The observed heating profile is consistent with the numerical calculations of the power deposition profile due to continuum Alfvén wave absorption. The $m / N=-1 /-2$ and 0/-3 AW continuum modes are dominant in the plasma core heating. In particular, the resonant absorption of the $m / N=0 /-3$ sideband harmonic plays an important role in the central core and $m / N=0 /-2$ mode in periphery plasma heating. 
Using the reflectometer with fixed frequency (32.3 $\mathrm{GHz}$ ), local density fluctuations with $4 \mathrm{MHz}$ frequency are found at local AW resonance. Theoretical analysis shows that the maxima of the detected phase shift are produced due to the local AW resonances of $m / N=-1 /-2$ and $1 /-3$ modes.

Finally, we can conclude that combination of AW excitation system with the ECE radiometry and the swap frequency reflectometer can be very power diagnostic tools to localize the AW power deposition, and to find effective mass number $A_{\text {eff }}$ and q-profiles in tokamak experiments.

\section{Acknowledgments}

We are thankful to our colleagues, participating in RF heating experiments in TCABR: J. I. Elizondo, A. N. Fagundes, Yu. K. Kuznetsov, I. C. Nascimento, E. M. Ozono, W. P. de Sá, E. A.O. Saettone, E. K. Sanada, J. H.F. Severo, O. C. Usuriaga, and A. Vannucci, and G.Amarante for collaboration. This work has been supported by the National Research Council (CNPq) of Brazil and the State of São Paulo Research Foundation (FAPESP).

\section{Appendix. Electron Cyclotron Emis- sion Radiometer}

A millimeter microwave detection system used for electron cyclotron emission measurements in the TCABR is a heterodyne sweeping radiometer based on a Backward Wave Oscillator (BWO), which operates in the frequency range of 52-85 GHz. The system can operate in two modes: fixed frequency (time resolution of $10 \mu \mathrm{s}$ ) and sweeping mode (50 $\mu$ s per step). The radiometer is calibrated in frequency and in radiation intensity. The frequency calibration is made by means of an precision harmonic oscillator $(54,60,66,72,78$ and $86 \mathrm{GHz}$ ). The absolute calibration was carried out using a blackbody (microwave absorber) immersed in liquid nitrogen $(77 \mathrm{~K})$ and also put in an oven with adjustable temperature up to $1470 \mathrm{~K}$. Two other components are also used for periodic intensity calibration check: a Cryogenic Matched Load and a Noise Source (time stability of .001dB/0C and flatness $+/-1.5 \mathrm{~dB}$ ). A mechanical waveguide switch and a $20 \mathrm{~dB}$ directional coupler are used to commute the radiometer from the measurement mode (coupled to the plasma) to the calibration mode. A Gaussian antenna is used for better space resolution measurements. Between the antenna and the radiometer, oversized waveguide are used to reduce the signal attenuation. The antenna axis is posed in the equatorial plane of the machine and perpendicular to the plasma column axis.

To use of ECE radiation for temperature measurements, the accessibility and absorption conditions must be taken into account. For a magnetic field of $B_{T 0}=1.15 \mathrm{~T}$, it was calculated and verified with density rise experiments in TCABR that the maximum permissible density to access the second harmonic in the $\mathrm{X}$ mode is $n_{e 0}=2.3 \times 10^{19} \mathrm{~m}^{-3}$. For the absorption condition of the black body radiation the most important parameter is the optical thickness. Using the optical thickness $(\tau)$ equation taken from Ref.[31], we calculate that the optical thickness condition $\tau \geq 1$ is satisfied in the TCABR plasma core, $-0.1 \mathrm{~m} \leq R-R_{0} \leq 0.1 \mathrm{~m}$.

\section{References}

[1] A.G. Elfimov, A.G. Kirov, and V.P. Sidorov, In High Frequency of Plasma Heating, edited by Litvak A G (Transl. Series, AIP, New York, 1992) p. 239.

[2] K. H. Burrell, Phys. Plasmas 4, 1499 (1997).

[3] H. Biglari, P. H. Diamond, and P. W. Terry, Phys. Fluids, B 2, 1 (1990).

[4] A.G. Elfimov, R.M.O. Galvão, I.C. Nascimento, and G. Amarante-Segundo, Plasma Phys. Controll. Fusion, 40, 451 (1998).

[5] V. V. Dolgopov and K. N. Stepanov, Nucl. Fusion5, 276 (1965).

[6] W. Grossmann and J. A. Tataronis, Z. Phys. 261, 217 (1973).

[7] A. Hasegawa and L. Chen, Phys. Rev. Lett. 35, 370 (1975).

[8] A. G. Elfimov, in: 2nd Joint Grenoble-Varenna Int. Symp. on Heating in Toroidal Plasmas, Como, Italy, 683, (1980).

[9] D. W. Ross, G. L. Chen, and S. M. Mahajan Phys. Fluids, 25, 652 (1982).

[10] K. Appert, R. Gruber, F. Troyon, and J. Vaclavik, Plasma Phys. 24, 1147 (1982).

[11] K. Itoh and S. I. Itoh, Plasma Phys. 25, 1037 (1983).

[12] I. J. Donnelly, B. E. Clang, and N. F. Craner, J. Plasma Phy$\operatorname{sics} 35,75$ (1986).

[13] J. Vaclavik and K. Appert, Nucl. Fusion, 31, 1945 (1991).

[14] B. Joye, A. Lietti, J. B. Lister, J.M. Moret, and W. Simm Phys. Rev. Lett. 56, 2481 (1986).

[15] G. A. Collins, F. Hofmann, B. Joye, R. Keller, A. Lietti, J.B. Lister, and A. Pochelon Phys. Fluids, 29, 2260 (1986).

[16] T.E. Evans, P.M. Valanju, J.F. Benesch, R. Bengston, Y. Li, S. Mahajan, M. Oaks, D. Ross, X. Wang, and J. Watkins Phys. Rev. Let., 53, 1743 (1984).

[17] T. Intrator, P. Probert, M. Vukovic, S. Wukitch, A.G. Elfimov, R. Durst, R.A. Breun, D. Brouchous, D. Diebold, M. Doszy, R. Fonk, N. Hershkowitz, M. Kishinevsky, C. Litwin, R. Majesky, and P. Nonn G. Winz Phys. Plasmas, 3, 1331 (1996).

[18] O. M. Shevts, I. A. Dikij, S. S. Kalinichenko, A. I. Lysojvan, N. I. Nazarov, T. Yu. Ranyuk, D. L. Grekov, K. N. Stepanov, and V. T. Tolok, Nucl. Fusion 26, 23 (1986).

[19] R. Bhen, G.A. Collins, J. B. Lister, and H. Weisen Plasma Phys. Contr. Fusion 29, 75 (1987).

[20] G. Amarante-Segundo, A. G. Elfimov, D. W. Ross, R. M.O. Galvo, and I. C. Nascimento, Phys. Plasmas, 6, 2437 (1999).

[21] S. A. Galkin, A. A. Ivanov, S. Yu. Medvedev, and A. G. Elfimov Comp. Phys. Communications, 143, 29-47, (2002).

[22] G.A. Collins, A.A. Howling, J. B. Lister, and Ph Marmillod, Plasma Phys. Contr. Fusion 29, 323 (1987).

[23] P. Descamps, G. Van Wassenhove, R. Koch, A.M. Messiaen, and P.E. Vandenplas Phys. Let. A 143, 311 (1990).

[24] J. B. Lister, L. Villard, and G. de Rider,: Plasma Phys. Contr. Fusion 40, 1635 (1998).

[25] L.F. Ruchko, E. Ozono, R.M.O. Galvão, I.C. Nascimento, F.T. Degasperi, and E.A. Lerche Fusion Engineering and Design 38, 15 (1998). 
[26] E.A. Lerche, E. Ozono, L.F. Ruchko, R. M. O. Galvão, A.G. Elfimov, Yu. K. Kuznetsov, I.C. Nascimento, A. N. Fagundes, W.P. de Sá, E. Sanada, J.I. Elizondo, D.O. Campos, R. Valencia, A. Vannucci, M.V.P. Heller, I.L. Caldas, V.P. Tsypin, A.A. Ferreira, E.A.Saetone, J.H.F. Severo, and V. Bellintani "Preliminary Results on Alfvén wave Heating in the TCABR Tokamak", AIP Conference Proc. 563, 191 (2001).

[27] A. G. Elfimov Phys. of Plasmas, 8, 2050 (2001).

[28] L. F. Ruchko, E. Lerche, R. M. O. Galvão, A.G. Elfimov, I.C. Nascimento, W.P. de Sá, E. Sanada, J.I. Elizondo, A.A Ferreira, E.A. Saetone, J.H.F. Severo, V. Bellintani, and O.N. Usuranga Braz. J. Phys., 32, 57 (2002).

[29] R. M. O. Galvão, V. Bellintani, Jr., A. G. Elfimov, A. N. Fagundes, A. M.M. Fonseca, Yu. K. Kuznetsov, E. A. Lerche, I. C. Nascimento, E.K. Ozono, L. F. Ruchko, and J. H.F. Severo, 30th EPS Conference on Plasma Phys. and Contr. Fu- sion, 7-11 July 2003, St.Pitersburg, Russia, ECA, 27A, (P4.50) (2003).

[30] L. F. Ruchko, R. Valencia, R. M. O. Galvão, E.A. Lerche, A.G. Elfimov, V. Bellintani, J.I. Elizondo, A.N. Fagundes, A.M.M. Fonseca, Y.K. Kuznetsov, I.C. Nascimento, W.P. de Sá, E. Sanada, and R.P. da Silva Rev. Scientific Instruments, 75, 655 (2004)

[31] M. Bornatici, R. Cano, O. De Barbieri, and F. Engelmann, Nuclear Fusion, 23, 1153 (1983).

[32] H. J. Hartfuss, T. Geist, and M. Hirsch, Plasma Phys. Contr. Fusion 39, 1693 (1997).

[33] V. L. Ginsburg, Propagation of Electromagnetic Waves in Plasmas (Gordon and Breach, New York, 1961).

[34] A.G. Elfimov Comments on Plasma Phys. and Contr. Nucl. Fusion, 17, 145 (1996) 